\title{
Diagnosis and Analysis of Explosive Cyclones Affecting the Safety of Ship Navigation
}

\author{
Guo Yana ${ }^{1, a^{*}}$, Ding Zhenguo ${ }^{1}$ \\ ${ }^{1}$ College of Navigation, Jiangsu Maritime Institute, Nanjing, Jiangsu, 211170, China \\ ${ }^{2}$ School of Education, Tianjin University, Tianjin, 300072, China \\ a email: gynxz@163.com \\ *corresponding author
}

Keywords: Explosive Cyclone, Weather Situation Analysis, Potential Vorticity, Eye Structure

\begin{abstract}
According to the final purpose analysis data provided by the National Environmental Prediction Center, the grid data of the second version of NCEP climate prediction system, and the infrared satellite cloud data provided by the tropical depression center of the United States, the explosive tropical storm occurred in the Northwest Pacific in January 2015. The evolution process and circulation of the system are analyzed. Using high resolution satellite cloud image data, the "eye" structure of the "spiral snake" distribution formed in the development of tropical storms is analyzed. The results show that there is a surface storm center in the front of $500 \mathrm{hPa}$ cyclone during the development of tropical storm. The strong positive vorticity flow and the explosion of atmospheric pressure develop. The divergence caused by high-altitude jet and low-level water vapor transport plays an important role in the explosive development of tropical storms. During the development of this tropical storm, the structure of tropical low pressure is similar to its "eyes", with "warm heart" structure and sinking moving area with eyes. In the first six hours when the tropical storm reaches maturity, the satellite cloud image appears as the center of the tropical storm. The cloud rotates inward several times in the eye to form a "spiral snake.". The distribution of the cloud system on the satellite cloud image is related to the horizontal distribution of the potential vorticity in the center of the high-altitude tropical storm. The cloud system is the reflection of the dynamic effect of the high-altitude vorticity.
\end{abstract}

\section{Introduction}

Explosive typhoons often occur in the sea and develop rapidly in a short time. With severe meteorological phenomena such as strong winds and rainstorms, they pose a great threat to the navigation of ships in the sea. Important academic value and application value. Yoshida and ASMA analyzed the structure of the explosive tropical storm in the Northwest Pacific, the position of the maximum deepening velocity of sea surface pressure, the moving path and the large-scale meteorological conditions. Explosive tropical storms are classified [1]. They point out that tropical storms often erupt in low-pressure areas. Low temperature advection and high-altitude eddy current are important factors for the explosive development of tropical storms. Meldi studied the meteorological situation of the explosive tropical storm and pointed out that the explosive tropical storm is related to the low level jet engine of $500 \mathrm{hPa}$. The predecessors did a lot of analysis on several famous tropical storms. For example, many scholars analyzed the "President's Day" tropical storm formed in the Atlantic Ocean in February 1979; gadam studied the zueen formed in the Atlantic Ocean by Hurricane Elizabeth in September 1978. A number of studies have shown that the high tilt angle and the instability of tilt pressure in the upper reaches are important factors for the development of explosive tropical storms. It is found that during the explosive development of several tropical storms, the "eye" structure will become more developed. This reminds me of the eye structure of a tropical cyclone [2]. A number of studies have shown that the tropical cyclone eye has a high altitude, warm heart structure and a sinking moving area. With regard to the 
morphological characteristics of the eyes of tropical cyclones, previous studies have pointed out that all the eyes of tropical cyclones are not regular circles or ellipses. Several tropical low-pressure "eyes" have formed "polygonal eyes" due to the asymmetric vortex structure in the tropical storm. Rand found that typhoons are found in the range of several times of the normal typhoon eye, and more studies also showed that several tropical cyclones formed two concentric "binoculars" in the development process. The explosive tropical storm occurred in the Northwest Pacific in January 2015 is analyzed. The purpose is to analyze the development, general circulation and physical structure of tropical storms.
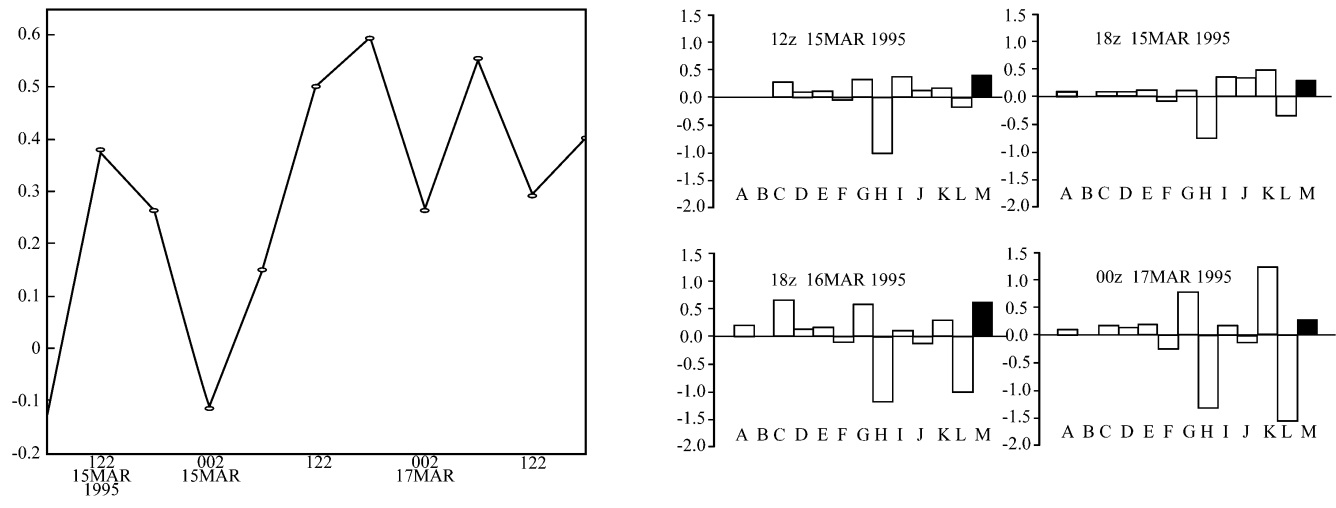

Figure 1 Relative vorticity of geostrophic cyclone center

\section{Data}

The data is the final purpose of the NCEP (final nalisis, FNL) grid data of the National Environmental Prediction Center. It analyzes the data that the horizontal grid spacing is, the vertical 26 layers are divided, the pressure surface, the time interval is 6 hours, that is, the earth, 612 earth, 18 earth, the potential development potential, temperature, longitude, wind speed, relative humidity and other variables, 98 is mainly used to analyze tropical storms and weather conditions. The download address [3]. In the second version of the grid data of DS83.2. NCEP climate prediction system CFSV 2 in UCAR database, the level of has lattice distance. It is vertically separated on 37 isobars, including formation height and air temperature. 127 variables were mainly used in the structural analysis of explosive eyes. The download address is http:RDA. UCAR data set. 3 hours MTSAT satellite infrared data from CIMSS, tropical cyclone center of the United States.

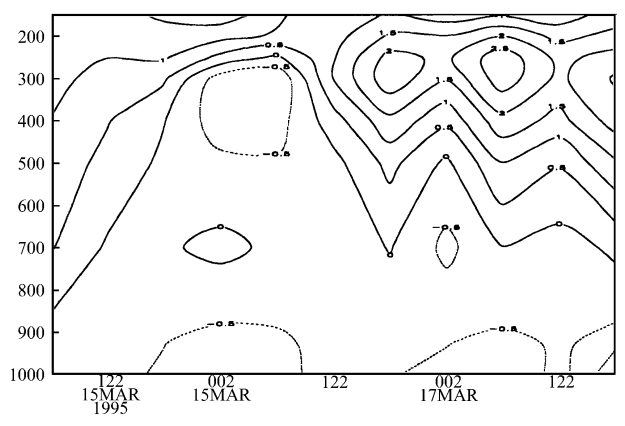

Figure 2 Vorticity advection term

\section{Evolution of Cyclones}

As shown in Figures 1 and 2, a tropical storm occurred near eastern Japan on January 2, 2015. In the next 24 hours, it moved rapidly to the northeast and the central air pressure continued to drop. 98bell 000 UTC is close to fourth, with a central pressure of 951.2 HPA, moving north and slowing down. On January 4, 2015, 18 UTC tropical storms began to move to the northwest, and the central air pressure continued to increase. It then peaked on January 5, 2015, near the southeast of the Kamchatka Peninsula. Figures 1 and 2 show the evolution of tropical storms[4]. The tropical storm 
was generated near eastern Japan on January 2, 2015 and moved rapidly to the Northeast within 24 hours. The central air pressure also continued to decrease. The maximum is 2.98 times the dragon. 000 UTC tropical storm is approaching the fourth, and the central pressure reaches 951.2 HPA, moving northward and slowing down. On January 4, 2015, 18 UTC tropical storms began to move northwest. The air pressure in the center continues to increase and the plug is inserted. That was on January 5, 2015, near the southeastern surface of the Kamchatka Peninsula. Descending stage: the tropical storm begins to weaken and be satisfied, and the central air pressure gradually increases. The tropical storm moved slowly to the northwest and rotated nearby. The cloud structure began to move, the outline began to blur and finally dissipated.

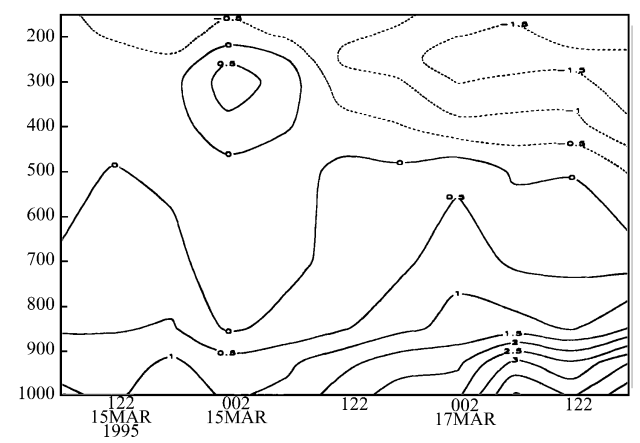

Figure 3 Horizontal dispersion of cyclone center in each stage of cyclone development

\section{Weather Situation Analysis}

This section analyzes the meteorological conditions for 300, 500, and 850 HPAs, as shown in Figures 4, 5, and 6. There are two short wave valleys in North and South Japan and a ridge facing Lake Baikal in the northeast of Japan. The north air stream in front of the ridge carries cold air from high latitude to the south[5]. Then, Japan's two shortwave trackers merged into a large East Asian valley. $850 \mathrm{hPa}$ shows that Japan is a cold circulation zone, which is conducive to the formation of Japan's frontal system and provides favorable conditions for the formation of surface tropical storms. In 300 HPA, two jet streams from the West and northwest of Japan are concentrated in Japan, forming a tropical storm type wind cone in Japan, which promotes the formation of surface tropical storms. The hurricane moved eastward and strengthened rapidly. Third, the pressure deepening speed of 12 UTC centers can reach up to 2.98 Bergren. In the terrain height field under $500 \mathrm{hPa}$, the track of East Asia moves eastward, indicating the southeast direction of Northwest[6]. In the northeast direction of Baikal Lake, more cold air is transported to the circulation, and the atmospheric pressure is further strengthened.

\section{Eye of Cyclone}

Tropical storms are in explosive development and clouds are comma shaped. Near the center of the cloud as the eye of a tropical storm, there is an area without obvious clouds. When the fourth UTC tropical storm reached maturity on the 4th the cloud formed an inward spiral structure in the "eye" area. After the development of tropical storm , the "eye" area of UTC tropical storm gradually expanded in 2009, and the internal multi cyclone spiral structure was not clear. The "eye" area of a tropical storm is shown in the box , which is formed by swirling cloud belts inside the tropical storm[7]. It is about $380 \mathrm{~km}$ long from east to west and $390 \mathrm{~km}$ long from north to south. The cloud belt rotates again inside the tropical storm, forming an "inner eye" about 100 kilometers in diameter. At the same time, the long and narrow cloudless zone extends anticlockwise from the southeast of the "eye" area. In the east of the "Mu" area, the width of the area without clouds is about 150 kilometers, while in the north of the "Mu" area, it is 80 kilometers. The width of the west side of the "eye" area is about $40 \mathrm{~km}$. This light and dark spiral structure looks like the shape of a "spiral snake."[8]. Tropical storm clouds are composed of water droplets and ice crystals, so precipitation can be used to diagnose the distribution of water vapor in tropical storms. After the tropical storm 
matures, its horizontal distribution also shows similar distribution characteristics to that of satellite cloud images. When the tropical storm reaches its mature stage, the clear multi Vortex Spiral eye structure first appears in the satellite cloud image. At this time, the potential vorticity field in the center of the tropical storm is still cluster like high frequency area. In the next 10 hours, the structural cloud system gradually slows down, and the image of swirling cloud field and satellite cloud gradually forms with the same compact structure, that is, the distribution of high potential vorticity is no longer the center concentration of tropical storm[9]. However, a spiral band is formed from the outside to the inside. In addition, when the high vorticity is concentrated in the center of the vorticity, the satellite cloud image shows an inward multiple helix structure due to the dynamic effect of the vorticity. With the development of the tropical storm, a large amount of dry air is introduced into the interior of the tropical storm, the vortex field forms a multi spiral structure, and the high potential vorticity is no longer concentrated in the center of the tropical storm. Therefore, the eye structure of satellite cloud image is difficult to maintain. That is to say, the special eye structure in the satellite cloud image may be the reflection of vorticity distribution at high altitude.

\section{Conclusion}

Tropical cyclones are often called "eye" areas. Because of their strong rotation, they form a round cloudless area in the center of tropical storms. When the fulminant tropical storm studied in this paper reaches maturity, the same "eye" region appears in the center of the tropical storm[10]. Tropical storm "eye wall" has a rising area. The "eye" area has a sinking area and a warming structure of the heart.

\section{Acknowledgements}

2019 Jiangsu Province Department of Education, Jiangsu Province Vocational College Teachers Professional Leaders high-end Research Project (project number 20190617;)2019 Jiangsu Provincial Department of Education, Jiangsu University Qinglan project Principal investigator, (project No. 20190304).

\section{References}

[1] WANG Shuai, FU Gang, PANG Huaji. Structure Analyses of the Explosive Extratropical Cyclone: A Case Study over the Northwestern Pacific in March, 2017.

[2] Difei Deng, Noel E. Davidson, Liang Hu,. Potential Vorticity Perspective of Vortex Structure Changes of Tropical Cyclone Bilis (2006) during a Heavy Rain Event following Landfall. Monthly Weather Review, vol. 145, no. 5, 2017.

[3] Tao Feng, Xin-yong Shen, Rong-hui Huang,. Influence of the interannual variation of crossequatorial flow on tropical cyclogenesis over the western North Pacific. Journal of Tropical Meteorology, vol. 23, no. 1, pp. 68-80, 2017.

[4] Peng D, Zhou T. Why was the arid and semiarid northwest China getting wetter in the recent decades?, vol. 122, 2017.

[5] Wernli H, Papritz L. Role of polar anticyclones and mid-latitude cyclones for Arctic summertime sea-ice melting, vol. 11, no. 2, 2018.

[6] LI Shanshan, LI Guoping. Evolution and Mechanism Analysis of a Plateau Vortex and Plateau Shear Line. Chinese Journal of Atmospheric Sciences, 2017.

[7] Lei Shi. An Object Detection and Pose Estimation Approach for Position Based Visual Servoing. Electrical Control \& Communication Engineering, vol. 12, no. 4, pp. 367-375, 2017.

[8] JEFF CALLAGHAN. ASYMMETRIC INNER CORE CONVECTION LEADING TO TROPICAL CYCLONE INTENSIFICATION. Tropical Cyclone Research and Review, no. z2, 
2017.

[9] Reason C J C. Variability in rainfall over tropical Australia during summer and relationships with the Bilybara High, vol. 132, pp. 1-14, 2017.

[10] Leslie M. Smith, Samuel N. Stechmann. Precipitating Quasi-Geostrophic Equations and Potential Vorticity Inversion with Phase Changes. Journal of the Atmospheric Sciences, vol. 74, no. 10, 2017. 